\title{
Is there a relationship between pain intensity and postural sway in patients with non-specific low back pain?
}

\author{
Alexander Ruhe ${ }^{1 *}$, René Fejer ${ }^{2+}$ and Bruce Walker ${ }^{3+}$
}

\begin{abstract}
Background: Increased center of pressure excursions are well documented in patients suffering from non-specific low back pain, whereby the altered postural sway includes both higher mean sway velocities and larger sway area. No investigation has been conducted to evaluate a relationship between pain intensity and postural sway in adults (aged 50 or less) with non-specific low back pain.

Methods: Seventy-seven patients with non-specific low back pain and a matching number of healthy controls were enrolled. Center of pressure parameters were measured by three static bipedal standing tasks of $90 \mathrm{sec}$ duration with eyes closed in narrow stance on a firm surface. The perceived pain intensity was assessed by a numeric rating scale (NRS-11), an equal number of patients $(n=11)$ was enrolled per pain score.
\end{abstract}

Results: Generally, our results confirmed increased postural instability in pain sufferers compared to healthy controls. In addition, regression analysis revealed a significant and linear increase in postural sway with higher pain ratings for all included COP parameters. Statistically significant changes in mean sway velocity in antero-posterior and medio-lateral direction and sway area were reached with an incremental change in NRS scores of two to three points.

Conclusions: COP mean velocity and sway area are closely related to self-reported pain scores. This relationship may be of clinical use as an objective monitoring tool for patients under treatment or rehabilitation.

\section{Background}

Increased postural sway is well documented in patients suffering from non-specific low back pain (NSLBP) [1] and a variety of theories exist regarding the effect of NSLBP on body sway. Postural control mechanisms are believed to be affected by damage to sensory tissues in the lumbar spine and trunk [2]. This deterioration of proprioceptive information reduces the accuracy of the sensory integration processes resulting in an imprecise estimation of the center of mass position [3], thereby inhibiting compensatory center of pressure (COP) shifts.

Acute "pain interference" [4] has also been proposed as a possible cause with discharge from high-threshold nociceptive afferents in the low back interfering with spinal motor-pathways [5] and the motor cortex [6]. In

\footnotetext{
* Correspondence: alexander_ruhe@hotmail.com

† Contributed equally

${ }^{1}$ Murdoch University, Praxis fuer Chiropraktik Wolfsburg, Wolfsburg, Germany Full list of author information is available at the end of the article
}

addition, pain may cause an increased pre-synaptic inhibition of muscle afferents [7] and affect the central modulation of proprioceptive spindles of muscles [8], thereby causing prolonged latencies by a decrease in muscle spindle feedback.

As outlined in our systematic literature review [1], several factors such as age [9-11], gender, weight [12], and height [13] have been shown to exhibit a significant effect on postural sway. The aim of this study is to investigate whether COP excursions are also affected by pain severity and pain duration and if so, to further describe this relationship. This relationship is worthy of investigation as it may show clinical significance for the application of COP measures.

To our knowledge, this is the first study to investigate this clinical question with a best practice experimental setup and also the first to comprehensively assess the relationship between pain and COP excursions over a

C Biomed Central

(c) 2011 Ruhe et al; licensee BioMed Central Ltd. This is an Open Access article distributed under the terms of the Creative Commons Attribution License (http://creativecommons.org/licenses/by/2.0), which permits unrestricted use, distribution, and reproduction in any medium, provided the original work is properly cited. 
wide spectrum of pain scores when compared to healthy controls.

\section{Methods \\ Subjects}

We aimed at enrolling around 80 participants for both symptomatic and control group. Previous sample size calculations for a group of controls and symptomatic patients with an NRS-11 score of $5.0 \pm 2.1$ using an Altman Nomogram [14] suggested recruitment of around 50 symptomatic and healthy participants each. We decided to exceed this number in order to compensate for potential dropouts.

All new patients entering a private chiropractic clinic in Wolfsburg, Germany were asked on the phone whether they would take part in this study. The healthy controls were friends and partners of already enrolled participants and were initially approached by them regarding the possibility of participation. If they displayed interest they were asked to contact the clinic for further details. After verbal and written information had been given, the subjects consented to participate in this study, which was approved by the Murdoch University Human Research Ethics Committee (Approval 2010/173).

The cut-off age for both controls and symptomatic individuals was 50 years, as after that age related impairments to postural stability could not be excluded [9-11].

Inclusion criteria for the symptomatic participants were NSLBP of any duration and the presence of pain $\geq$ 2 on the NRS-11 scale on the day of the postural sway recordings. Participants were excluded if the pain went below the gluteal fold, there were positive nerve root findings, serious spinal deformities, any condition that might affect balance (e.g. whiplash associated disorder or vestibular pathologies) or previous significant injuries such as traumatic damage to the spine or spinal surgery. No pain medication was allowed within 24 hours prior to the recordings. Participants were also excluded if they were unable to perform the postural sway recording either due to pain or other reasons. We aimed at enrolling around 10 patients for the 9 pain intensity groups (NRS 2-10).

For the purpose of this study, healthy was defined as the absence of any self-reported neurological or musculoskeletal impairments, pain or disability for a minimum of 6 months prior to the time of evaluation. Specifically, individuals with a history of low back pain within 6 months or previous injury to the neck or lower extremities, any known balance problems or the usage of medication associated with pain suppression or altered sensory perception were excluded. The physical examination of the control group must also have ruled out any back or extremity complaints or significant biomechanical impairments that might influence the measurements.

\section{Procedures}

Prior to the COP measurements, a physical examination was conducted on all participants by two experienced and trained chiropractors (TB and AS) who were otherwise not involved in the study. This procedure aimed to assess whether the volunteers met the criteria for their respective group and met the physical demands of the study. The NSLBP participants were further asked to describe their pain intensity at the time of recording by means of an NRS-11, a rating scale ranging from 0 (no pain) to 10 (worst possible pain) [15].

The experimental setup was based on an earlier literature review where a best practice setup for obtaining reliable COP data was published [16]. Accordingly, trials were conducted with eyes closed as the data obtained shows higher reliability than with eyes open. We further considered that the loss of visual input would prove an additional challenge to the balance system. In this way deficits in proprioception may be more easily detected and the discriminative value of the measurement between healthy controls and symptomatic NSLBP participants enhanced.

The system used for this study was a Metitur Good Balance GB300 ${ }^{\circledR}$ CE (Metitur Oy, Finland). Signals were sampled at $100 \mathrm{~Hz}$, amplified and converted from analogue to digital. High frequency noise was reduced by a low-pass filter with a cut-off frequency of $10 \mathrm{~Hz}$.

Mean velocity ( $\mathrm{mVel}$ ) was chosen as the main COP parameter as this has consistently shown to be both reliable [16] and discriminative for NSLBP [1]. It is described by taking the total distance of the COP path travelled in the respective direction and dividing it by the sampling duration. In addition, the $90 \%$ circle diameter was included to offer a broader spectrum of analysis. This parameter refers to the diameter of a circle containing $90 \%$ of the COP path travelled over a given time.

The participants were asked to remove their shoes and stand upright on the forceplate with their eyes closed, the head erect and their arms hanging loosely by their sides. The foot position was narrow stance with toes and heels touching. For the duration of the recording, the participants were further instructed to "stand as still as possible" [17].

Three successive trials of 90 seconds duration each were conducted with a preceding $5 \mathrm{sec}$ adaption period that was not recorded. Rest periods of $60 \mathrm{sec}$ were provided between each trial during which the participants were allowed to sit down while maintaining their original foot position on the forceplate. All participants were 
asked afterwards whether pain or discomfort may have influenced their balance performance.

All tests were conducted in a quiet room with normal temperature. The forceplate was calibrated prior to the recordings and further underwent an automatic calibration check before each trial.

\section{Data analysis \\ Age effects}

To test if postural sway is influenced by age [9-11], the healthy participants were subdivided into two age ranges (20-35 and 36-50 years) and subsequently compared to see if they statistically differ from each other. If, however, our study showed no significant differences, the age groups were to be combined for further analysis to reduce the risk of type-II error.

\section{Reliability}

To test the reliability of the COP measures for this experimental setup for both controls and pain sufferers, the two-way random-effect intra-class correlation coefficient $(\mathrm{ICC} 2, \mathrm{k})$ as described by Shrout and Fleiss [18] was computed using absolute agreement. For the purpose of this study it was interpreted using the following criteria: 0.0-0.39 poor, 0.40-0.59 fair, 0.60-0.74 good and 0.75-1.00 excellent [19]. In addition, the $95 \%$ confidence intervals (CI) and the standard error of measurement (SEM) [20] were calculated.

\section{Relationship between pain intensity and postural sway}

We also tested the assumptions of homogeneity of variance (Levene statistic) and normality, where ShapiroWilk test was conducted for all independent variables and the dependent variables separately per pain group. The COP data was further analyzed using the GamesHowell test. Means, standard deviations (SDs) and 95\% confidence intervals (CIs) were calculated for all dependent variables.

Stepwise univariate regression analysis was conducted to assess for the possible effect of each of the following variables: age, gender, weight, height, pain intensity and previous pain duration on COP $\mathrm{mVel}$ and $90 \%$ circle diameter. This was followed by a multivariate regression analysis where independent variables that showed a significant effect during univariate analysis were included.

To investigate the appropriate form of regression analysis, the SPSS Curve Estimation function was applied to scatter plots for variables stated above (independent variables) and the COP parameters (dependent variables). In addition, collinearity diagnostics were applied. The level of statistical significance was set at $p \leq 0.05$.

All data were exported to PASW ${ }^{\circledR}$ Statistics 18 (SPSS Inc, 2009) for statistical analysis.

\section{Results}

\section{Subjects}

Eighty-two individuals suffering from NSLBP initially volunteered to participate in this study. We did not reach our target number of at least 10 NSLBP participants for NRS scores $9(n=2)$ and $10(n=0)$ and therefore only included NRS scores 2-8 with 11 NSLBP participants each. Five symptomatic participants were excluded as they exhibited severe pain $(n=4)$ or an antalgic posture $(n=1)$ when standing and were unable to complete the tests. This left a total of 77 NSLBP sufferers (37 females, $45 \%$ ) to which a matching number of healthy controls were enrolled. All participants were able to complete the trials without difficulty and did not report increased pain or discomfort during the COP recordings. The characteristics of the participants are shown in Table 1.

\section{Age groups}

Both age groups had a similar number of healthy participants with $n=36$ for $18-35$ yrs and $n=41$ for $36-50$ yrs. As there was no statistically significant difference in COP measures between the two groups (Table 2), the data were combined and analyzed for the control group as a whole.

\section{Reliability}

With three recordings being averaged from the both healthy controls and symptomatic participants, the included COP parameters reached good reliability throughout (Table 3).

Table 1 Demographic and functional characteristics

\begin{tabular}{|c|c|c|c|c|c|c|}
\hline & $\begin{array}{l}\text { NSLBP } \\
\text { Age 20-35 } \\
(\mathrm{n}=32)\end{array}$ & $\begin{array}{l}\text { Healthy controls } \\
\text { Age 20-35 } \\
(\mathrm{n}=36)\end{array}$ & $\begin{array}{c}\text { Statistical difference } \\
\text { p-value }\end{array}$ & $\begin{array}{l}\text { NSLBP } \\
\text { Age 36-50 } \\
(\mathrm{n}=45)\end{array}$ & $\begin{array}{l}\text { Healthy } \\
\text { controls } \\
\text { Age } 36-50 \\
(\mathrm{n}=41)\end{array}$ & $\begin{array}{c}\text { Statistical } \\
\text { Difference } \\
\text { p-value }\end{array}$ \\
\hline Age (years) & $28.9 \pm 4.7$ & $29.8 \pm 4.4$ & 0.89 & $44.1 \pm 4.3$ & $43.5 \pm 5.5$ & 0.67 \\
\hline Height (cm) & $178.0 \pm 6.6$ & $177.2 \pm 7.4$ & 0.36 & $179.2 \pm 7.6$ & $176.9 \pm 6.9$ & 0.37 \\
\hline Weight (kg) & $77.6 \pm 9.5$ & $77.3 \pm 11.7$ & 0.47 & $80.8 \pm 12.8$ & $76.9 \pm 8.8$ & 0.71 \\
\hline BMl & $24.3 \pm 2.7$ & $24.9 \pm 3.9$ & 0.60 & $25.1 \pm 2.9$ & $24.5 \pm 1.9$ & 0.11 \\
\hline NRS-11 (0-10) & $4.9 \pm 1.9$ & N/A & N/A & $5.1 \pm 2.1$ & N/A & N/A \\
\hline Previous pain duration (weeks) & $19.9 \pm 33.6$ & N/A & N/A & $18.7 \pm 30.5$ & N/A & N/A \\
\hline
\end{tabular}

Values are mean \pm SD 
Table 2 Comparison of COP data between the age groups

\begin{tabular}{cccc}
\hline COP parameter & $\begin{array}{c}\text { Healthy controls } \\
\mathbf{2 0 - 3 5} \text { yrs }(\mathbf{n}=\mathbf{3 6})\end{array}$ & $\begin{array}{c}\text { Healthy controls } \\
\mathbf{3 6 - 5 0} \text { yrs }(\mathbf{n}=\mathbf{4 1})\end{array}$ & $\begin{array}{c}\text { Statistical difference } \\
\mathbf{p} \text {-value }\end{array}$ \\
\hline $\mathrm{mVel} \mathrm{ML}(\mathrm{mm} / \mathrm{s})$ & $11.8 \pm 2.5$ & $12.0 \pm 2.7$ & 0.28 \\
$\mathrm{mVel} \mathrm{AP}(\mathrm{mm} / \mathrm{s})$ & $9.1 \pm 2.7$ & $9.5 \pm 2.1$ & 0.27 \\
$90 \%$ circle diameter $(\mathrm{mm})$ & $11.6 \pm 2.8$ & $12.0 \pm 2.4$ & 0.21 \\
\hline
\end{tabular}

Values are mean $\pm S D$

AP: antero-posterior, mVel: mean velocity, ML: medio-lateral

\section{Relationship between pain intensity and postural sway} As a general trend, a steady linear increase in mVel AP/ ML and 95\% circle diameter direction can be observed. Levene's Tests showed no homogeneity of variance ( $p \leq$ 0.018 ) while Shapiro-Wilk test indicated a normal distribution of the independent and dependent variables $(\mathrm{p} \geq$ $0.11)$.

Compared to healthy controls, a significant difference $(p \leq 0.01)$ in $\mathrm{mVel}$ was present in NSLBP participants beginning at an NRS score of 3 in ML direction. In AP direction, statistical significance $(p \leq 0.05)$ was also reached at a pain intensity of 3 with an increase in significance from 5 to $8(p \leq 0.001)$ (Figure 1$)$.

Compared to healthy controls, a significant difference in $90 \%$ circle diameter was only present at NRS scores of 6,7 and $8(p \leq 0.001)$ (Figure 2$)$.

The differences in postural sway between pain scores as assessed by Games-Howell are presented in Tables 4 and 5. With regards to $\mathrm{mVel}$ differences between the individual pain scores, significance was reached at lower NRS scores in ML compared to AP direction (Table 4).

Finally, the relative differences between pain scores for the parameter $90 \%$ circle diameter are demonstrated in Table 5 . The same trend as seen with mean sway velocity can be observed. However, at pain intensities 2 and 3 , significant differences between pain scores are present at larger intervals (3 NRS scores compared to 1-2 at mVel ML/AP).

\section{Regression analysis}

The SPSS Curve Estimation function showed that a linear relationship was the most suitable line of fit $(\mathrm{p} \leq$ 0.001). Hence, linear regression was used for further analyses of the data. No co-linearity between the variables was determined.

Table 3 Reliability of COP measures

\begin{tabular}{ccccccc}
\hline COP parameter & \multicolumn{3}{c}{ NSLBP $(\mathbf{n}=\mathbf{7 7})$} & \multicolumn{3}{c}{ Healthy controls $(\mathbf{n}=$} \\
& & & & & $\mathbf{7 7})$ \\
\cline { 2 - 7 } & $\mathbf{I C C 2 , \mathbf { k }}$ & $\mathbf{9 5 \% C l}$ & SEM & $\mathbf{I C C 2 , k}$ & $\mathbf{9 5 \% C l}$ & SEM \\
\hline mVel ML & 0.85 & $0.79-0.99$ & 0.96 & 0.89 & $0.73-0.97$ & 0.89 \\
mVel AP & 0.83 & $0.76-0.88$ & 0.86 & 0.85 & $0.63-0.96$ & 0.96 \\
90\% circle diameter & 0.71 & $0.61-0.79$ & 1.29 & 0.69 & $0.57-0.77$ & 1.44 \\
\hline
\end{tabular}

AP: antero-posterior, mVel: mean velocity, ML: medio-lateral
The univariate regression analysis included the variables gender, age, weight, height, previous pain duration and pain intensity. With the exception of previous pain duration, all other independent variables exhibited a significant effect on $\mathrm{mVel} \mathrm{AP} / \mathrm{ML}$ and $90 \%$ circle diameter and were consequently included in the multivariate analysis. This further analysis showed that only pain intensity exhibited a significant effect on the selected COP parameters.

For mean velocity and pain intensity, the regression analysis was a reasonably good fit, describing $53.0 \%$ of the variance in $\mathrm{mVel} \mathrm{ML}$ and $40.0 \%$ in mVel AP $\left(\mathrm{R}^{2}\right.$ adj $=51.0 \%$ and $\mathrm{R}^{2}$ adj $=38.4 \%$ respectively), the overall relationship was highly significant in both $\mathrm{ML}$ and AP direction $(\mathrm{F}=40.8, p<0.001$ and $\mathrm{F}=24.9, p<0.001$ respectively). Mean sway velocity increased by $1.53 \mathrm{~mm} /$ $\mathrm{s}$ for every extra pain level in $\mathrm{ML}$, and by $1.27 \mathrm{~mm} / \mathrm{s}$ for every extra pain level in AP direction.

The regression analysis for the parameter $90 \%$ circle diameter and pain intensity was a poor fit, describing just $18.7 \%$ of the variance in circle diameter $\left(R^{2}\right.$ adj $=$ $16.5 \%)$. The overall relationship, however, was highly significant $(\mathrm{F}=8.6, p<0.001)$. The $90 \%$ circle diameter of the COP excursion increased by $0.6 \mathrm{~mm}$ for every extra pain level.

\section{Discussion}

We were unable to enroll a sufficient number of NSLBP participants for all pain intensity groups to allow analysis of all 10 NRS scores. This may be explained by the fact that patients with NRS scores of 9 and higher are not commonly encountered in a chiropractic practice as the potential severity of the condition warrants medical attention instead.

We were able to demonstrate a linear relationship between pain intensity and postural sway velocities in both AP and ML direction as well as for the parameter 90\% circle diameter. This is in agreement with a general observation by Lihavainen et al. [21] who conducted a similar study in a geriatric population. They did not, however, investigate postural sway related to the individual pain scores but reached their conclusions based on a subdivision into mild or moderate/severe pain only.

Even though an increased sway velocity started at a lower pain score in the AP direction, the overall 


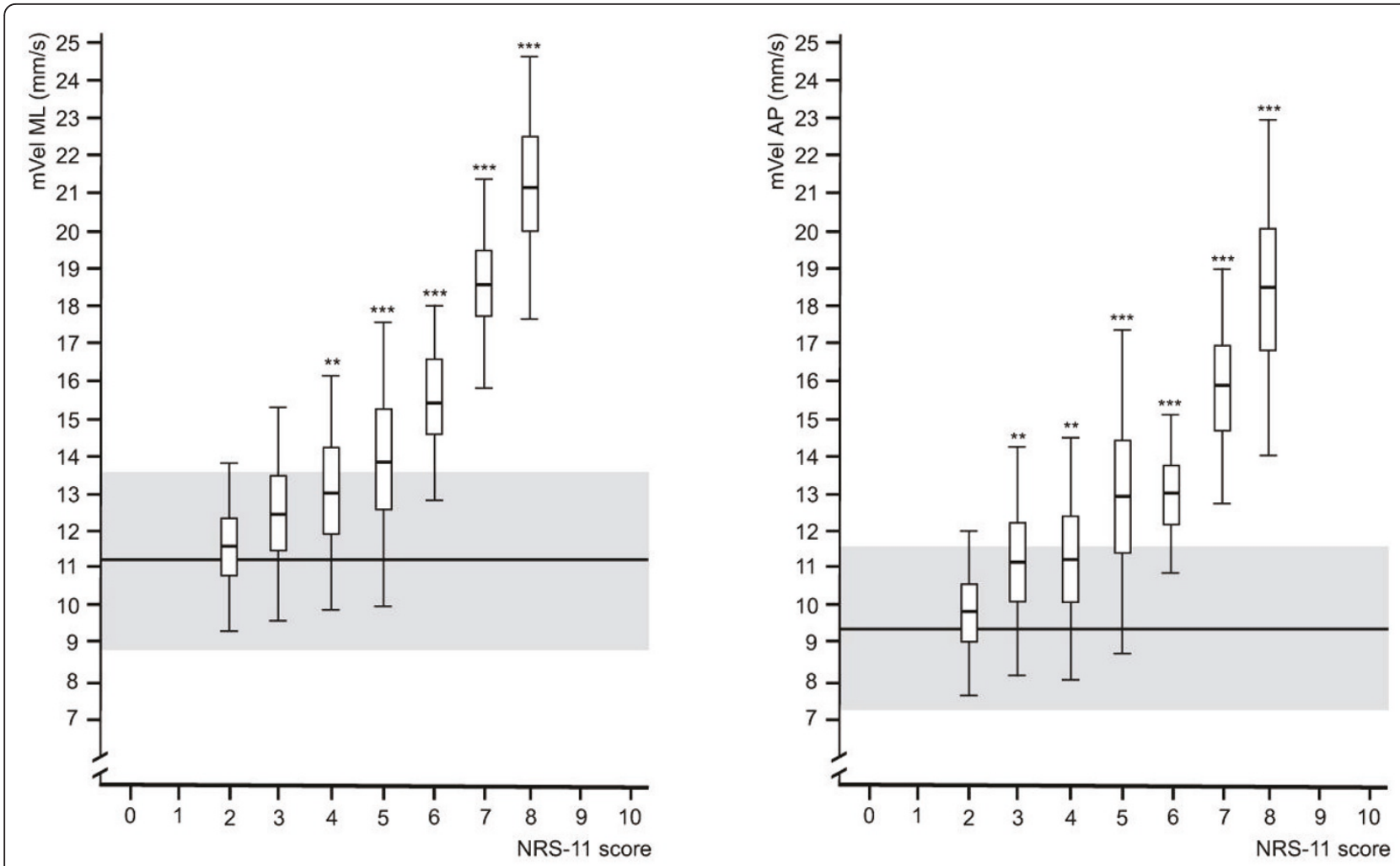

Figure 1 Relationship between pain intensity and mean sway velocity in AP and ML. The horizontal line and the grey area indicate the mean score of healthy controls and the standard deviations respectively. The vertical lines indicate standard deviations; the boxes show mean and $95 \% \mathrm{Cls}$ respectively. Levels of significance compared to controls: ${ }^{*} p \leq 0.05,{ }^{* *} p \leq 0.01,{ }^{* * *} p \leq 0.001$

difference compared to healthy controls was similar to that in the ML direction. On the other hand, the ML sway velocity increased at a faster rate. In addition, this study confirms the altered postural sway characteristics previously reported in a systematic review of NSLBP sufferers [1]. The review noted higher COP mVel values (particularly the AP direction) and a larger sway area compared to healthy controls was described.

The non-overlapping 95\% CIs associated with NRS scores at higher pain intensities, particularly with mVel $\mathrm{AP} / \mathrm{ML}$, are surprising and may be attributable to our standardized experimental setup and selection of participants. Such a clear subdivision appears unlikely at first sight due to the inherently varying pain perception between individuals.

As the $90 \%$ circle diameter is exclusively used with the Metitur system, it is not possible to put the respective results into context. However, it corresponds to the various parameters applied in the literature to describe COP sway area and may therefore offer at least limited comparability.

Our data, however, does not allow for an explanation of the underlying mechanism of the observed pain associated alterations in COP sway velocity. However, as previous pain duration did not exhibit a significant effect on postural sway as pain intensity has, this may suggest that pain interference [4] may be the determining factor. Neuro-physiological changes, on the other hand, are rather dependent on pain duration and therefore a significant time effect would have been expected. Future studies assessing postural sway before and after acute pain stimulation or using analgesics in chronic and acute NSLBP participants may add valuable information in this respect.

Furthermore, as no other studies have looked into the relationship between a broader range of pain intensities and COP measures it is not possible to compare our results.

At lower and medium pain intensities there was no apparent change in the COP parameters. This may be due to participants finding it difficult to decide on their "true" score, NRS-5 for example shows the widest standard deviations across all parameters. This may therefore explain why no statistically significant differences were observed between lower pain scores (NRS 2-4) for most parameters and may account at least partially for the variability in the associated COP measurements. However, as the confidence intervals across all pain 


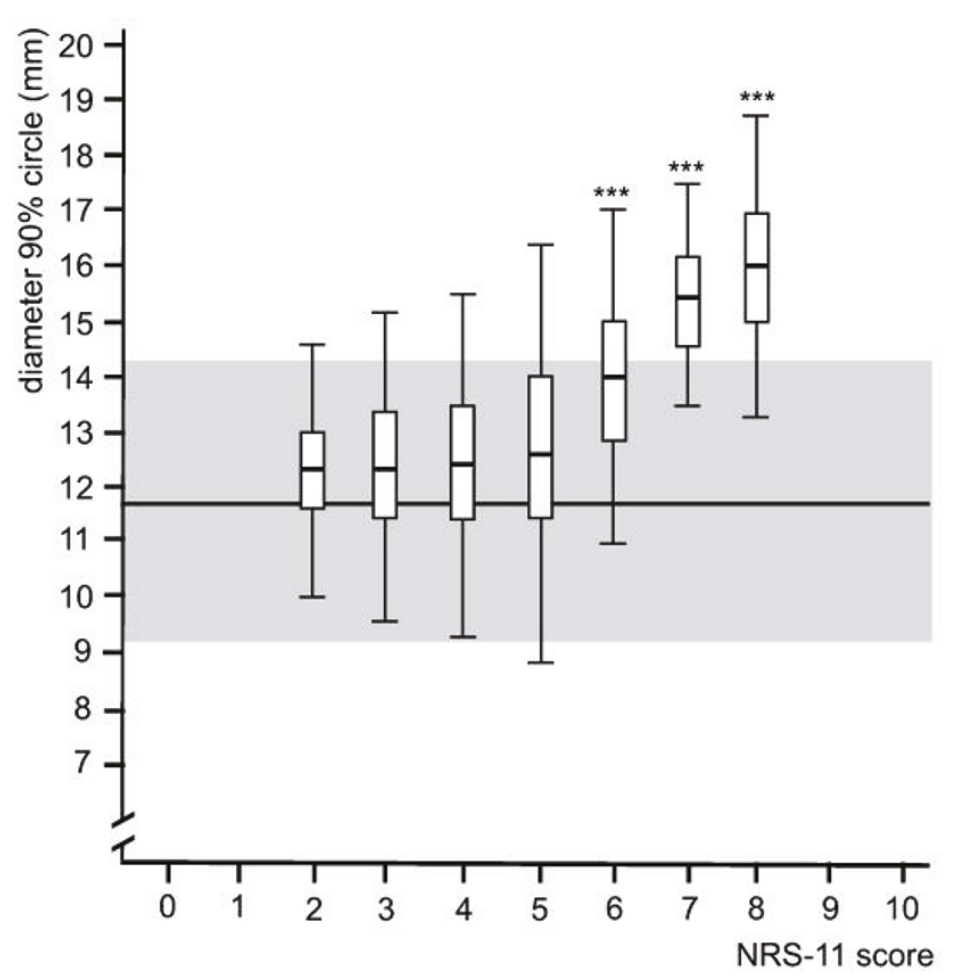

Figure 2 Relationship between pain intensity and $\mathbf{9 0 \%}$ circle diameter. The horizontal line and the grey area indicate the mean score of healthy controls and the standard deviations respectively. The vertical lines indicate standard deviations; the boxes show mean and $95 \% \mathrm{Cls}$ respectively. Levels of significance compared to controls: * $p \leq 0.05,{ }^{* *} p \leq 0.01,{ }^{* * *} p \leq 0.001$.

scores remain fairly consistent, the variability of the postural sway measurements most likely reflects individual variations within the COP excursions. The results also suggest that the neurological alteration previously described [4-8] may only have an impact on COP measures at medium to high intensities (i.e. NRS $\geq 5$ ).

\section{Table 4 Sway differences between NSLBP participants and pain free controls using NRS-11 scores for mVel AP and ML}

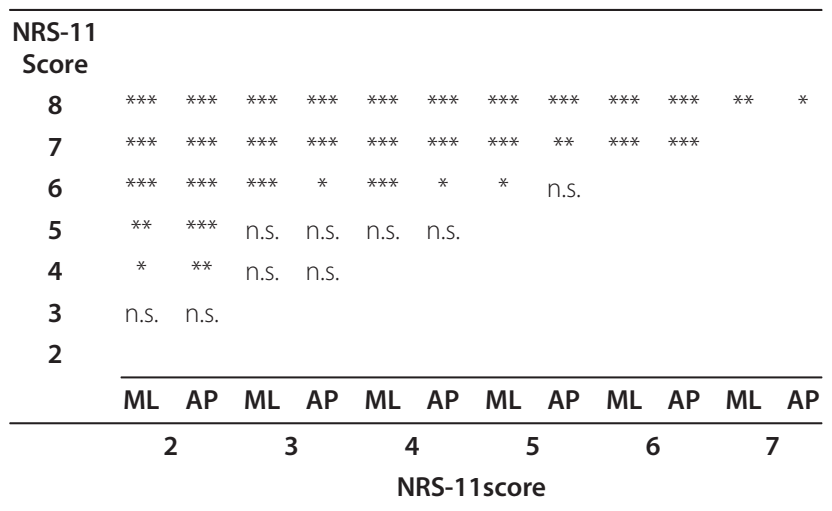

n.s.: not significant $(p>0.05)$

Levels of significance: ${ }^{*} p \leq 0.05,{ }^{* *} p \leq 0.01,{ }^{* * *} p \leq 0.001$
In contrast to other studies [9-13], we could not demonstrate any significant effect of age, height, weight or gender on COP excursions in the patient group. This may be attributed to the demographics and physical characteristics of the participants as well as our COP measurement protocol. Our results were derived using a protocol based on best evidence [16], nevertheless future studies are needed to confirm these findings using the same protocol.

Table 5 Sway differences between NSLBP participants and pain free controls using NRS-11 scores for $90 \%$ circle diameter

\begin{tabular}{ccccccc}
\hline 8 & $* * *$ & $* * *$ & $* * *$ & $* *$ & $*$ & n.s. \\
7 & $* * *$ & $* * *$ & $* * *$ & $* *$ & $*$ & \\
6 & $*$ & n.s. & $*$ & n.s. & & \\
5 & n.s. & n.s. & n.s. & & & \\
4 & n.s. & n.s. & & & & \\
3 & n.s. & & & & & \\
2 & & & & & & \\
\hline $\begin{array}{c}\text { NRS-11 } \\
\text { Score }\end{array}$ & 2 & 3 & 4 & 5 & 6 & 7 \\
\hline
\end{tabular}

n.s.: not significant $(p>0.05)$

Levels of significance: ${ }^{*} p \leq 0.05,{ }^{* *} p \leq 0.01,{ }^{* * *} p \leq 0.001$ 


\section{Clinical considerations}

The COP measurement protocol used in this study may in future be suitable as an objective outcome measure for clinical monitoring purposes. However, the results are unidirectional in that increasing pain was associated with increasing postural sway. We have not established that decreasing pain leads to a decreasing postural sway.

Secondly, given the linear relationship between pain intensity and, for example, $\mathrm{mVel}$, a clinically significant decrease of two points on a pain NRS [20] is equivalent to a reduction in mean sway velocity of $3.6 \mathrm{~mm} / \mathrm{s}$ in $\mathrm{ML}$ and of $3.0 \mathrm{~mm} / \mathrm{s}$ in AP direction. These changes lie between 1 and 2 standard deviations from the mean. It remains to be seen if such a reduction is also clinically significant.

In addition, this study indicates that any future sample size calculations for COP measurements involving pain sufferers should be considered in the light of the respective perceived intensity. Depending on the research purpose, the inclusion criteria may focus on those with NRS-scores of 5 or higher to reach significance compared to controls more readily.

The results may also cast a new light on the interpretation of studies that reported no significant differences in postural sway between symptomatic individuals and healthy controls. In those instances (e.g. Brumange et al. [22] and Mok et al. [23]), these observations may be attributable to the low perceived pain intensities of the NSLBP participants enrolled.

There is evidence that higher COP sway is associated with a higher risk of falling in the elderly [24] and sustaining injuries as a consequence, although this is subject to debate $[25,26]$. Our results did not include geriatric participants and therefore cannot be generalized to that population, however our data may nevertheless underline the importance of suitable pain control in elderly pain sufferers to avoid falls.

In addition, as pain interference appears a likely underlying mechanism, the focus of a rehabilitative approach in pain sufferers with increased COP excursions should be on pain reduction rather than proprioceptive training.

Future studies may also show a role for COP measurements as part of a suite of other procedures to identify malingerers. Even if the individual is aware that pain is associated with greater COP excursions, a study with pseudo-malingerers showed that imitating pain related sway pattern is difficult at best and the average results for sway velocity and sway area greatly exceeded those expected from a real pain sufferer [27].

\section{Strengths and Limitations}

The major strength of this study is in its best practice experimental setup which ensured reliable data collection. Our inclusion and exclusion criteria further prohibited our overall results from being affected by demographic or anthropometric factors.

In this cross-sectional study the subjective nature of pain perception and therefore pain rating may have influenced the results. In addition, pain perception between younger and older NSLBP participants varies and a decrease in pain perception in geriatric individuals has been described [28]. Although this does not affect our sample groups with a cut-off age of 50 yrs, it nevertheless prohibits our results to be generalized to elderly patients.

While significant differences in postural sway compared to healthy controls could be demonstrated in our patient population, the overall number of participants per NRS score was still comparably small. Our results are therefore prone to be affected by extreme COP measures. Other sample groups with identical NRS scores may therefore show varying results. However, we expect the linear trend to be preserved. Similar studies with an identical experimental setup and larger sample sizes should be conducted to confirm our results.

\section{Conclusions}

Despite the subjective nature of pain perception and the unclear causative factors, the results of this study show that in adults (18 and 50 years) with NSLBP, increasing COP sway velocity increases linearly with increasing perceived pain intensity greater than 4 on an NRS scale. This trend, while less obvious, is also apparent for the parameter $90 \%$ circle diameter.

\section{Acknowledgements}

We would like to thank Tino Bos for his valuable contribution by conduction the physical examinations and the clinic staff for their continuous support in recruiting the participants and organizing measurement appointments.

\section{Author details}

${ }^{1}$ Murdoch University, Praxis fuer Chiropraktik Wolfsburg, Wolfsburg, Germany. ${ }^{2}$ Chiropractor, Research Department, Spine Centre of Southern Denmark, Hospital Lillebaelt and University of Southern Denmark, Middelfart, Denmark. ${ }^{3}$ School of Chiropractic and Sports Science, Murdoch University, Murdoch, Western Australia, Australia.

\section{Authors' contributions}

AR carried out the COP measurements, conducted the statistical analysis and drafted the manuscript. RF and BW participated in the study design and assisted in drafting the manuscript. All authors read and approved the final manuscript.

\section{Competing interests}

The authors declare that they have no competing interests.

Received: 18 April 2011 Accepted: 15 July 2011 Published: 15 July 2011

\section{References}

1. Ruhe A, Fejer R, Walker B: Center of pressure excursion as a measure of balance performance in patients with non-specific low back pain compared to healthy controls: a systematic review of the literature. European Spine Journal: official publication of the European Spine Society, the 
European Spinal Deformity Society, and the European Section of the Cervical Spine Research Society 2011, 20:358-368.

2. Brumagne $S$, Cordo P, Lysens R, Verschueren S, Swinnen S: The role of paraspinal muscle spindles in lumbosacral position sense in individuals with and without low back pain. Spine 2000, 25:989-994.

3. Casadio M, Morasso PG, Sanguineti V: Direct measurement of ankle stiffness during quiet standing: implications for control modelling and clinical application. Gait Posture 2005, 21:410-424.

4. Moseley GL, Hodges PW: Are the changes in postural control associated with low back pain caused by pain interference? The Clinical Journal of Pain 2005, 21:323-329.

5. Rossi A, Decchi B, Ginanneschi F: Presynaptic excitability changes of group la fibres to muscle nociceptive stimulation in humans. Brain Research 1999, 818:12-22.

6. Rossi S, della Volpe R, Ginanneschi F, Ulivelli M, Bartalini S, Spidalieri R, Rossi A: Early somatosensory processing during tonic muscle pain in humans: relation to loss of proprioception and motor 'defensive' strategies. Clinical neurophysiology: official journal of the International Federation of Clinical Neurophysiology 2003, 114:1351-1358.

7. Sibley KM, Carpenter MG, Perry JC, Frank JS: Effects of postural anxiety on the soleus H-reflex. Human movement science 2007, 26:103-112.

8. Capra NF, Ro JY: Experimental muscle pain produces central modulation of proprioceptive signals arising from jaw muscle spindles. Pain 2000, 86:151-162.

9. Doyle TL, Dugan EL, Humphries B, Newton RU: Discriminating between elderly and young using a fractal dimension analysis of centre of pressure. Int J Med Sci 2004, 1:11-20.

10. Hageman PA, Leibowitz JM, Blanke D: Age and gender effects on postural control measures. Arch Phys Med Rehabil 1995, 76:961-965.

11. Demura S, Kitabayashi T, Noda M, Aoki H: Age-stage differences in body sway during a static upright posture based on sway factors and relative accumulation of power frequency. Percept Mot Skills 2008, 107:89-98.

12. Hue O, Simoneau M, Marcotte J, Berrigan F, Dore J, Marceau P, Marceau S, Tremblay A, Teasdale N: Body weight is a strong predictor of postural stability. Gait Posture 2007, 26:32-38.

13. Chiari L, Rocchi L, Cappello A: Stabilometric parameters are affected by anthropometry and foot placement. Clin Biomech (Bristol, Avon) 2002, 17:666-677.

14. Altman DG: Statistics in Practice London: British Medical Association; 1982.

15. Jensen MP, Karoly P, Braver S: The measurement of clinical pain intensity: a comparison of six methods. Pain 1986, 27:117-126.

16. Ruhe A, Fejer $R$, Walker BF: The test-retest reliability of centre of pressure measures in bipedal static task conditions - A systematic review of the literature. Gait Posture 2010, 32:436-445.

17. Zok M, Mazza C, Cappozzo A: Should the instructions issued to the subject in traditional static posturography be standardised? Medical Engineering \& Physics 2008, 30:913-916.

18. Shrout PE, Fleiss JL: Intraclass correlations: uses in assessing rater reliability. Psychological Bulletin 1979, 86:420-428.

19. Cicchetti DV, Sparrow SA: Developing criteria for establishing interrater reliability of specific items: applications to assessment of adaptive behavior. Am J Ment Defic 1981, 86:127-137.

20. Weir JP: Quantifying test-retest reliability using the intraclass correlation coefficient and the SEM. J Strength Cond Res 2005, 19:231-240.

21. Lihavainen K, Sipila S, Rantanen T, Sihvonen S, Sulkava R, Hartikainen S: Contribution of musculoskeletal pain to postural balance in communitydwelling people aged 75 years and older. J Gerontol A Biol Sci Med Sci 2010, 65:990-996.

22. Brumagne S, Janssens L, Knapen S, Claeys K, Suuden-Johanson E: Persons with recurrent low back pain exhibit a rigid postural control strategy. European spine journal: official publication of the European Spine Society, the European Spinal Deformity Society, and the European Section of the Cervical Spine Research Society 2008, 17:1177-1184

23. Mok NW, Brauer SG, Hodges PW: Hip strategy for balance control in quiet standing is reduced in people with low back pain. Spine 2004, 29 E107-112.

24. Pajala S, Era P, Koskenvuo M, Kaprio J, Tormakangas T, Rantanen T: Force platform balance measures as predictors of indoor and outdoor falls in community-dwelling women aged 63-76 years. J Gerontol A Biol Sci Med Sci 2008, 63:171-178
25. Brauer SG, Burns YR, Galley P: A prospective study of laboratory and clinical measures of postural stability to predict community-dwelling fallers. J Gerontol A Biol Sci Med Sci 2000, 55:M469-476.

26. Sihvonen SE, Sipila S, Era PA: Changes in postural balance in frail elderly women during a 4-week visual feedback training: a randomized controlled trial. Gerontology 2004, 50:87-95.

27. Endo K, Suzuki H, Yamamoto K: Consciously postural sway and cervical vertigo after whiplash injury. Spine 2008, 33:E539-542.

28. Gibson SJ, Helme RD: Age-related differences in pain perception and report. Clin Geriatr Med 2001, 17:433-456, v-vi.

\section{Pre-publication history}

The pre-publication history for this paper can be accessed here: http://www.biomedcentral.com/1471-2474/12/162/prepub

doi:10.1186/1471-2474-12-162

Cite this article as: Ruhe et al:: Is there a relationship between pain intensity and postural sway in patients with non-specific low back pain? BMC Musculoskeletal Disorders 2011 12:162.

\section{Submit your next manuscript to BioMed Central and take full advantage of:}

- Convenient online submission

- Thorough peer review

- No space constraints or color figure charges

- Immediate publication on acceptance

- Inclusion in PubMed, CAS, Scopus and Google Scholar

- Research which is freely available for redistribution 